\title{
Complete Response of Metastatic Melanoma to Second Line Chemotherapy with Paclitaxel and Carboplatin - Case Report
}

\author{
Krešimir Tomić, Ivana Tica Sedlar, Gordana Berić Jozić, Inga Marijanović, \\ Blanka Lukić, Dragana Miletić, Ana Puljić Ćuk
}

\author{
Clinic of Oncology, University Clinical \\ Hospital Mostar, Mostar, Bosnia and \\ Herzegovina \\ Correspondence: \\ kresotomic3@gmail.com \\ Tel.: + 38736336466 \\ Fax.: + 38736336442
}

Received: 19 October 2017

Accepted: 21 March 2018

Key words: Metastatic melanoma Chemotherapy - Second line.

\section{Introduction}

Melanoma is an aggressive tumour with the advanced disease characterized by widespread metastatic lesions, and the tumour has traditionally been resistant to most forms of treatment. Median survival for metastatic disease is only 6 - 9 months, a 1 -year survival rate of $25 \%$, and a 3 year survival of $15 \%$ (1).

The incidence of malignant melanoma in Europe varies from $19.2 \%$ in Switzerland to $2.2 \%$ in Greece per 100,000 persons/year (2). Most patients with metastatic melanoma need systemic treatment. Metastatic melanoma is considered to be a chemotherapy resistant disease so the role of chemotherapy in stage IV is less significant. Two distinct therapeutic modalities, tyrosine kinase inhibitors and immunotherapy might improve response rate, progression free survival and overall survival for metastatic melanoma (1). In many transition countries, such as Bosnia and Herzegovina, institutional barriers and the poor socioeconomic situation make it difficult to provide cancer treatment according to contemporary guidelines, and we are forced to treat patients with the available chemotherapy drugs.

In this case report we showed that chemotherapy can achieve excellent results in the treatment of metastatic melanoma.

\section{Case report}

A 57-year old female patient with ECOG (Eastern Cooperative Oncology Group) performance status 0 , was diagnosed with malignant melanoma of the trunk in July 2011. In terms of comorbidities, she had arterial hypertension, atrial fibrillation, coxarthrosis and gonarthrosis of the left knee. 


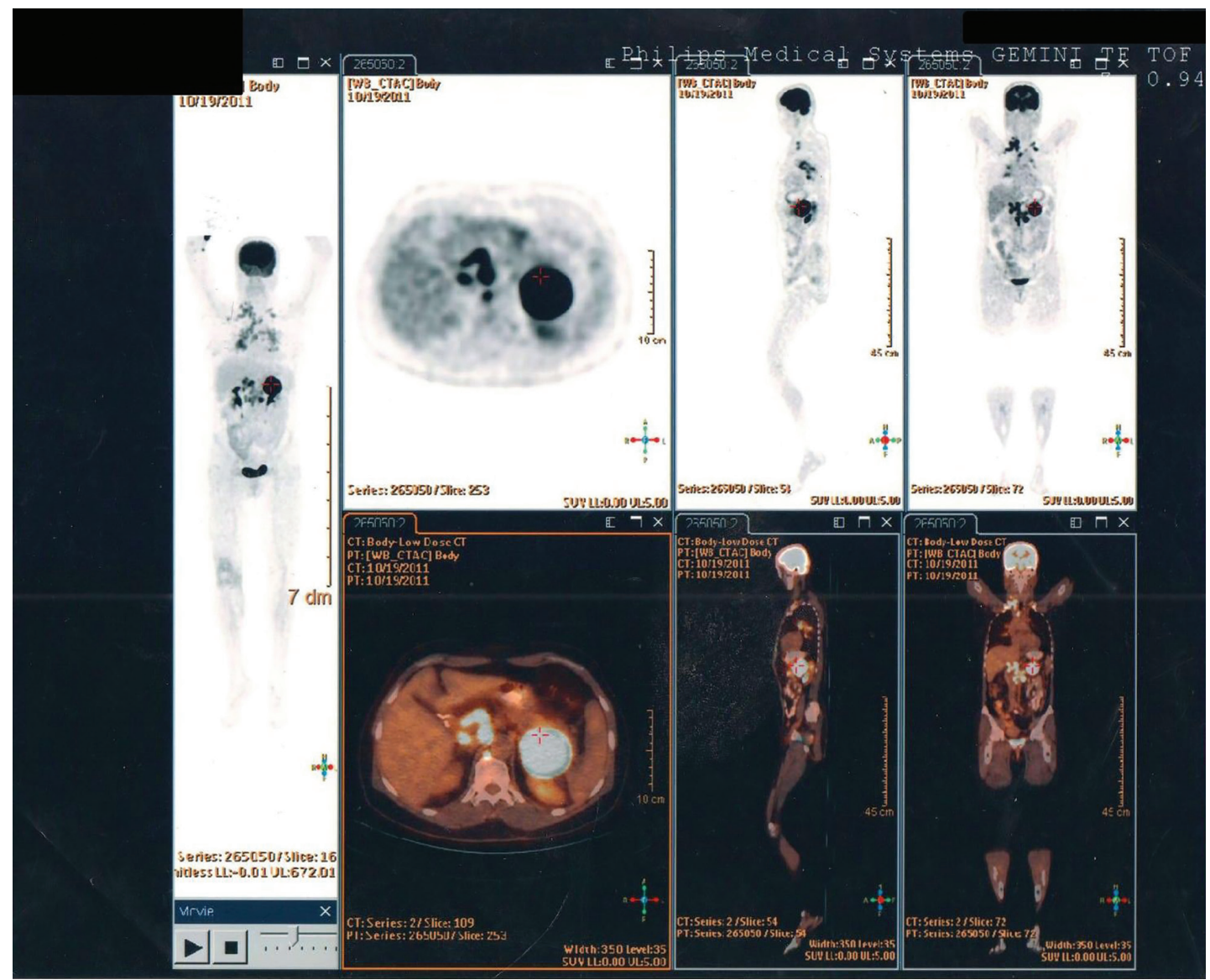

Figure 1.PET/CT scan; pathological metabolism of glucose in the lymph nodes of neck, mediastinum and abdomen, lesions in the pulmonary parenchyma and left adrenal gland.

The pathology report after surgery showed a malignant melanoma, Clark 4, Breslow 4. BRAF V600E analysis was not initially performed, but subsequent analysis confirmed BRAF V600E mutation. Postoperative PET/ CT in October 2011 detected metastatic melanoma in the lymph nodes of the neck, mediastinum, abdomen, and lesions in the pulmonary parenchyma and the left adrenal gland (Figure 1).

The serum LDH level was within reference range. Fine needle aspiration cytology (FNAC) of the lymph node at the neck did not show malignant cells. At the end of October 2011, chemotherapy was initiated with dacarbazine $(250 \mathrm{mg} / \mathrm{m} 2 /$ day $)$ and it was given intravenously for 5 days every 4 weeks. After 3 cycles of chemotherapy, control CT scans showed the stable disease by RECIST criteria, but after 7 cycles of chemotherapy control PET/CT showed progression of the disease with newly discovered metastases in the bones. From the side effects of dacarbazine chemotherapy the patient had grade 1 anaemia and grade 1 nausea. Chemotherapy with dacarbazine was discontinued due to the disease progression in May 2012, and we then started second line chemo therapy treatment with paclitaxel $(175 \mathrm{mg} / \mathrm{m} 2)$ and carboplatin (AUC 4) (TC regimen) intravenously every 3 weeks. Bisphosphonate Zolendronic acid was administered every 4 weeks because of the bone metastasis. Regular follow up with MSCT scans and PET/ $\mathrm{CT}$ was performed during treatment. Due to peripheral neuropathy grade 3 we reduced 

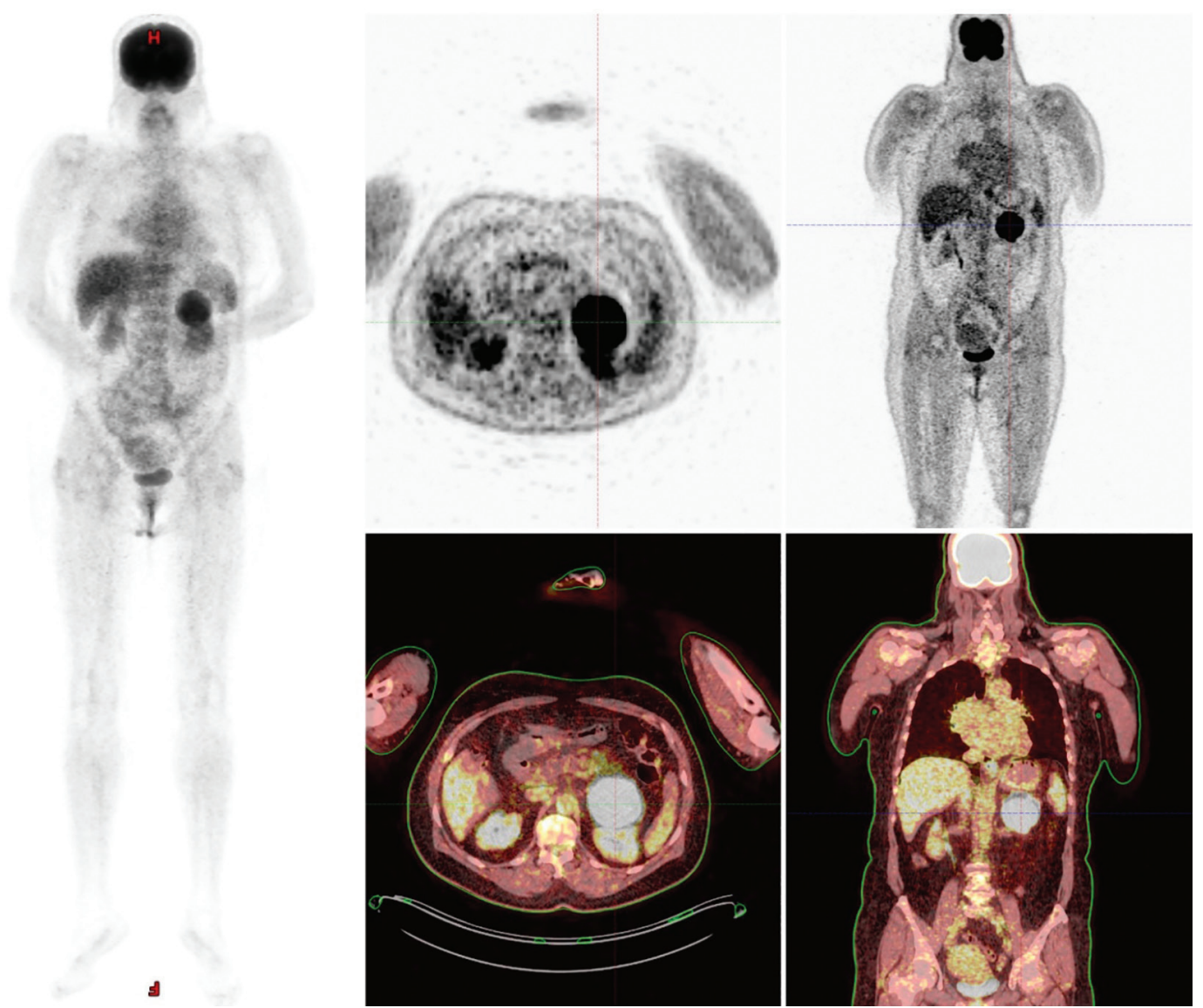

Figure 2. PET/CT scan; pathological metabolism only in left adrenal gland.

the dose of paclitaxel by $20 \%$ after 11 cycles of chemotherapy. Other side effects were grade 2 leukopenia, grade 2 granulocytopenia, grade 1 anaemia, grade 1 hypokalaemia and grade 1 hypocalcaemia. After 22 cycles of chemotherapy, in January 2014, control PET/CT showed complete disease response with a stable lesion by RECIST criteria in the left adrenal gland (Figure 2).

In March 2014, left adrenalectomy was performed and the pathology report showed a 5 centimeter benign granulocyte cell tumour. After that, a control CT scan showed no evidence of disease, so we discontinued all oncological treatment and continued regular follow-up. The last oncologist examination was in January 2016. The patient was in good general condition and the control CT scan showed no evidence of disease. In April
2016 we found by heteroanamnesis that the performance status of the patient was poor, and from the accompanying medical documentation we could see that the patient had suspected disease progression on the pleura, liver, kidney and tumour formation with heart compression. The patient died in May 2016 , and the cause of death was probably related to disease progression.

\section{Discussion}

We report complete response to second line chemotherapy with TC regimen in a patient with metastatic melanoma. The treatment goal for metastatic melanoma patients is to prolong overall survival, reduce tumourrelated symptoms and maintain quality of life. The treatment of metastatic melanoma 
has undergone huge changes in the last few years. Before the era of new therapeutic options, we treated patients with chemotherapy, such as dacarbazine, temozolamide, paclitaxel, albumin-bound paclitaxel, and carboplatin/paclitaxel or docetaxel (3).

According to previous data, dacarbazine showed an improved objective response rate (ORR), median progression free survival (PFS) and overall survival (OS) $(4,5)$. Our patient had PFS for 5.5 months with Dacarbazine, the best treatment response was a stable disease with side effects such as grade 1 anaemia and grade 1 nausea, which was similar to the reported side effects in the trials with Dacarbazine $(4,5)$. There have been two phase 2 studies that showed positive results with taxane and carboplatin in the second line treatment of metastatic melanoma $(6,7)$, with one complete response with docetaxel and carboplatin (7). Also there have been two phase 3 studies that showed no statistically significant difference in PFS, OS and ORR, when comparing the paclitaxel and carboplatin regimen (TC) with or without sorafenib in first and second line metastatic melanoma $(8,9)$. In study by Pflugfelder A, et al. (10) which compared the effectiveness of the TC regimen in the first and second line treatment of metastatic melanoma, the results were equivalent in PFS, OS and response.

Here, we present an isolated case of a patient with extensive chemotherapy duration and complete response to a paclitaxel and carboplatin regimen. The optimal duration of chemotherapy in metastatic melanoma has not been defined. In clinical practice we continue chemotherapy treatment until disease progression and/or unacceptable toxicities. In comparison to the other studies, it is important to notice that our patient had a complete response after 22 cycles of second line chemotherapy with the TC regimen (6, $8,10)$. In the study by Lee CK, et al. (7) there was one complete response to second line chemotherapy with docetaxel and carboplatin, which is similar to our results with paclitaxel and carboplatin. Due to grade 3 peripheral neuropathy during treatment with the second line TC regimen, we reduced the dose of paclitaxel by $20 \%$ after 11 cycles of chemotherapy. Other side effects were grade 2 leukopenia, grade 2 granulocytopenia, grade 1 anaemia, grade 1 hypokalaemia and grade 1 hypocalcaemia. Our patient's adverse events corresponded to the adverse events verified in the phase 2 and 3 studies $(6,8,9,7)$.

For a BRAF V600E mutated metastatic melanoma, targeted therapy with tyrosine kinase inhibitors vemurafenib and dabrafenib showed significantly better results in first line treatment, compared with Dacarbazine $(11,12)$. The combination of vemurafenib and cobimetinib compared to vemurafenib has statistically significantly greater PFS and ORR, at the cost of increased toxicity (13), and the combination of dabrafenib and trametinib, compared to dabrafenib, has statistically significantly greater PFS and OS (14).

Immunotherapy is a new therapeutic option that has shown excellent results in the treatment of patients with metastatic melanoma. The anti PD-1 antibodies nivolumab and pembrolizumab, anti CTLA antibodies ipilimumab and the combination of nivolumab and ipilimumab are immunotherapy options used in metastatic melanoma patients (3). A recently published phase 3 study showed the superiority of nivolumab and ipilimumab compared to monotherapy with nivolumab or ipilimumab. The median OS was not reached in the combined group, versus 37.6 months in the nivolumab group, which was statistically significant compared to 20 months with ipilimumab after 36 months of monitoring (15). The overall survival (OS) of our patient was 55 months, which was a significant result, comparable to the results of new therapeutic options, and significant in comparison to survival 
achieved by chemotherapy in randomized clinical trials $(11-14,15)$.

Since Bosnia and Herzegovina belongs to the group of countries with low socioeconomic development, new therapeutic options are unavailable because of their high prices. According to the recently published study by Kandolf-Sekulovic, et al. (16), more than 5000 patients in Europe do not have access to the recommended first line therapy for treatment of metastatic melanoma. With the lack of new therapeutic options, such as immunotherapy and targeted therapy, our patient case report shows that there are isolated cases where excellent therapeutic results can be achieved with the available therapeutic options, such as paclitaxel and carboplatin.

\section{Conclusion}

Treatment of metastatic melanoma with chemotherapy has inferior results compared to new therapeutic options, including tyrosine kinase inhibitors and immunotherapy. Due to the unavailability of new therapeutic options, chemotherapy represents the only available therapeutic modality for most patients in Bosnia and Herzegovina. Our case report showed that chemotherapy can achieve excellent results in the treatment of metastatic melanoma and this case could be interesting for other countries with similar local situation and not only for Bosnia and Herzegovina.

\footnotetext{
What is already known on this topic

Standard treatments of metastatic melanoma according to oncology guidelines are tyrosine kinase inhibitors and immunotherapy. Chemotherapy produces modest results in the treatment of metastatic melanoma.
}

\section{What this study adds}

Due to the unavailability of new therapeutic options, chemotherapy represents the only available therapeutic modality for most patients in Bosnia and Herzegovina. We report complete response in our patient which is similar to study by Lee CK, et al. about the potential clinical activity of second line com- bination treatment with taxane and carboplatin in metastatic melanoma (7).

Authors' contributions: Conception and design KT and ITS; Acquisition, analysis and interpretation of data: KT, ITS, BL, IM, GBJ, DM, and APĆ; Drafting the article: KT and ITS; Revising it critically for important intellectual content: KT, ITS and GBJ; Approved final version of the manuscript: KT, ITS and DM.

Conflict of interest: The authors declare that they have no conflict of interest.

\section{References}

1. Eggermont AM, Spatz A, Robert C. Cutaneous melanoma. Lancet. 2014;383(9919):816-27.

2. Forsea AM, Del Marmol V, de Vries E, Bailey EE, Geller AC. Melanoma incidence and mortality in Europe: new estimates, persistent disparities. $\mathrm{Br} J$ Dermatol. 2012;167(5):124-30.

3. NCCN.org/professionals/physician/pdf/melanoma. Washington: [updated 2018 Jan 18; cited 2018 March 14]. Avaible from: https://www.nccn.org/ about/contact.aspx.

4. Middleton MR, Grob JJ, Aaronson N, Fierlbeck G, Tilgen W, Seiter S, et al. Randomized phase III study of temozolomide versus dacarbazine in the treatment of patients with advanced metastatic malignant melanoma. J Clin Oncol. 2000;18(1):158-66.

5. Chapman PB, Einhorn LH, Meyers ML, Saxman S, Destro AN, Panageas KS, et al. Phase III multicenter randomized trial of the Dartmouth regimen versus dacarbazine in patients with metastatic melanoma. J Clin Oncol. 1999;17(9):2745-51.

6. Rao RD, Holtan SG, Ingle JN, Croghan GA, Kottschade LA, CreaganET, et al. Combination of paclitaxel and carboplatin as second-line therapy for patients with metastatic melanoma. Cancer. 2006;106(2):375-82.

7. Lee CK, Jung M, Choi HJ, Kim HR, Kim HS, Roh MR, et al. Results of a Phase II Study to Evaluate the Efficacy of Docetaxel and Carboplatin in Metastatic Malignant Melanoma Patients Who Failed First-Line Therapy Containing Dacarbazine. Cancer Res Treat. 2015;47(4):781-9.

8. Hauschild A, Agarwala SS, Trefzer U, Hogg D, Robert C, Hersey P, et al. Results of a phase III, randomized, placebo-controlled study of sorafenib in combination with carboplatin and paclitaxel as second-line treatment in patients with unresectable stage III or stage IV melanoma. J Clin Oncol. 2009;27(17): 2823-30. 
9. Flaherty KT, Lee SJ, Zhao F, Schuchter LM, Flaherty L, Kefford R, et al. Phase III trial of carboplatin and paclitaxel with or without sorafenib in metastatic melanoma. J Clin Oncol. 2013;20;31(3):373-9.

10. Pflugfelder A, Eigentler TK, Keim U, Weide B, Leiter U, Ikenberg $\mathrm{K}$, et al. Effectiveness of carboplatin and paclitaxel as first- and second-line treatment in 61 patients with metastatic melanoma. Plos One. 2011;16;6(2):e16882.

11. Chapman PB, Hauschild A, Robert C, Haanen JB, Ascierto P, Larkin J, et al. Improved Survival with Vemurafenib in Melanoma with BRAF V600E Mutation. N Engl J Med. 2011;364:2507-16.

12. Hauschild A, Grob JJ, Demidov LV, Jouary T, Gutzmer R, Millward M, et al. Dabrafenib in BRAF-mutated metastatic melanoma: a multicentre, open-label, phase 3 randomised controlled trial. Lancet. 2012;380(9839):358-65.
13. Larkin J, Ascierto PA, Dréno B, Atkinson V, Liszaky G, Maio M, et al. Combined vemurafenib and comitenib in BRAF-mutated melanoma. N Engl J Med. 2014;371(20):1867-76.

14. Long GV, Stroyakovskiy D, Gogas H, Leychenko E, de Braud F, Larkin J, et al. Dabrafenib and trametinib versus dabrafenib and placebo for Val600 BRAF-mutant melanoma: a multicentre, doubleblind, phase 3 randomised controlled trial. Lancet. 2015;386(9992):444-51.

15. Wolchok JD, Chiarion-Sileni V, Gonzalez R, Rutkowski P, Grob JJ, Cowey CL, et al. Overall Survival with Combined Nivolumab and Ipilimumab in Advanced Melanoma. N Engl J Med. 2017;377(14):1345-56.

16. Kandolf Sekulovic L, Peris K, Hauschild A, Stratigos A, Grob JJ, Nathan P, et al. More than 5000 patients with metastatic melanoma in Europe per year do not have access to recommended first-line innovative treatments. Eur J Cancer. 2017;75:313-22. 